\title{
MINERAL PARTICLE ROTATION MEASUREMENTS FOR MAGNETIC ROTATION SEPARATION
}

\author{
N.R. ALLEN \\ Ka (Pty) Ltd., 14 Station Lane, Exton, Tasmania 7303, Australia
}

(Received 28 September 2001; In final form 14 December 2001)

The quantities of magnetic susceptibility or magnetic moment can be used to determine which minerals can be separated from what other minerals by magnetic attraction separation. Now that magnetic rotation separation has been shown to be both possible and practical, there is a need for a practical quantity to describe relative mineral rotation strengths.

Such "rotation strength" quantities already exist in mineral physics, in forms such as magnetocrystalline and shape anisotropy constants (or fields). Although these quantities can be used as a guide to mineral particle rotational response to a rotating magnetic field, they are not simple to understand or easy and cheap to measure for very small individual particles, and do not include the effects of other dynamic magnetisation factors such as domain wall velocities. Nor do they include particle inertia and particle shape effects that are of practical concern in a magnetic rotation separation.

A practical quantity, the rotation index, can be defined in such a way that it includes all of the above factors and more accurately predicts particle response in a magnetic rotation separator. The rotation index is easily measured on individual particles as small as $50 \mu \mathrm{m}$, with the same equipment used for magnetic rotation separation.

Measurements of rotation index on a population of particles can reveal much about the ferromagnetic structures within the particles, and, used with measurements of magnetic susceptibility, can predict the results of combinations of attraction and rotation magnetic separations.

Keywords: Magnetic susceptibilityMagnetic field; Rotation index

\section{INTRODUCTION}

The rotation of mineral particles during a magnetic separation has been used probably as long as magnetic separation has existed. In 
many cases this rotation is more a side-effect of separator construction than a deliberate attempt to rotate the particles. For example, magnetic flocs in a low intensity wet drum separator are rotated slowly as they are carried past successive magnetic poles, and as they do so they release at least some of the entrapped nonmagnetic particles. As early as 1920 some inventors were patenting electromagnetic rotating filed separators that were designed to use particle rotation to decrease nonmagnetic or nonmetallic entrapment, but none of these appears to have made its mark on the mineral processing industry.

However, the rotation of a magnetized element in an external field was well known to physics before this. Maxwell, in his "A Treatise on Electricity and Magnetism" (1873) [1] describes the mathematics involved in the rotation of a magnetic element in a magnetic field, and also describes delicate instruments to measure it that are very similar to more modern ones for the measurement of magnetic anisotropy. Maxwell does not mention the term "magnetic anisotropy", and did not know the detailed theory behind it, but the concept and the basic mathematics were all there by 1873 . Magnetic particle rotation in a rotating magnetic field is really a very old idea. It is surprising that so little has been made of it since.

Magnetic anisotropy refers to the force that directs magnetisation along certain directions within a ferromagnetic crystal, known as directions of easy magnetisation. A direction of easy magnetisation is a lowenergy direction for magnetic alignment within a crystal. If an external magnetic field is directed at an angle to a direction of easy magnetisation in a particle, a torque is placed on the particle and the particle will attempt to rotate so as to line up its magnetisation with that of the external field. The maximum torque is applied to a particle when the angle between the external field and a direction of easy magnetisation is a quarter of the angle to the next direction of easy magnetisation.

\section{THE ROTATION INDEX}

The rotation index is based on the sine of the angle $(\phi)$ between particle magnetisation and external field that produces the greatest torque on a particle in a rotating magnetic field. This is smaller that the angle between the external field and a direction of easy 
magnetisation, because the particle magnetisation will actually be deflected (the amount depending on the anisotropy field) by the external field. The measured value will not directly give the sine of $\phi$ because of factors such as particle inertia and, if the rotating field is close enough to the critical field, domain wall velocity. For a particle with the anisotropy field much greater than the external field, the relationship between the factors can be expressed as

$$
R_{f}=\frac{\sin \left(\phi+D_{w}\right)}{1+I}
$$

where $R_{f}$ is the rotation index; $D_{w}$ is the domain wall velocity factor; $I$ is the inertia factor and $\phi$ is the angle between particle magnetisation and external field.

The practical measurement and calculation, as described below, assumes that the particle is rotating on a surface and has a cubic shape. If the particle is more rounded, for example, it will rotate easier, and give a higher rotation index that it should. Therefore, Eq. (1), for the measurement method used here, needs to include a "roundness factor":

$$
R_{f}=\frac{r \times \sin \left(\phi+D_{w}\right)}{1+I}
$$

\section{THE APPROXIMATE MEASUREMENT OF PARTICLE SUSCEPTIBILITY AND ROTATION INDEX}

Measurements are made in a rotating magnetic field for both susceptibility and rotation. Figure 1 shows the RMF laboratory separator set up for particle measurement. This separator would normally be used for routine laboratory magnetic separation work, but if the launders are removed it can double as a particle magnetic measurement machine.

Particles are loaded into individual water-filled glass tubes. The water gives the particles some buoyancy and dampens erratic motion. Two different measurements are made on each particle, at each desired 


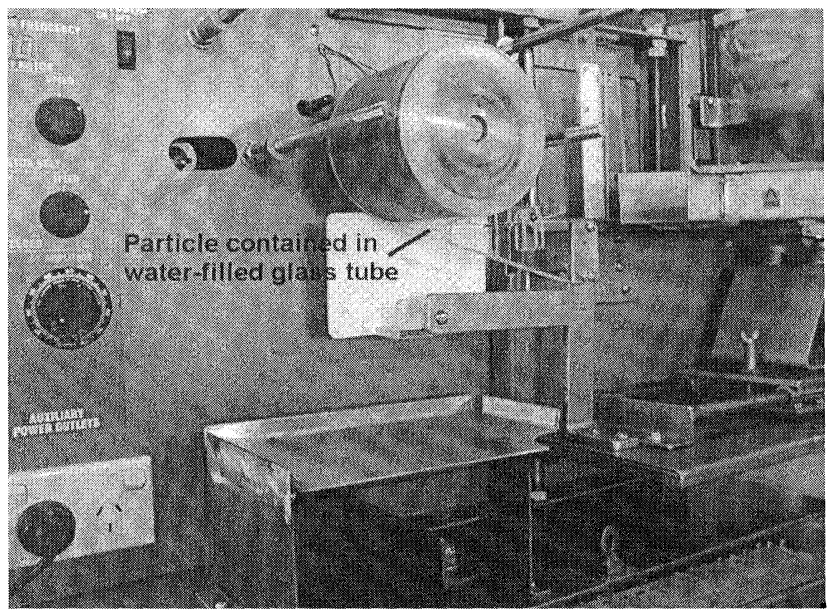

FIGURE 1 The Ka laboratory separator set up for particle magnetic measurements.

field rotation frequency. Beginning with the particle well below the magnet rotor, in a field not sufficient to either rotate it or lift it, the particle is raised towards the rotor until it just commences continuous rotation. The field and gradient at which this occurs can be calculated from the distance below the rotor. The particle is then moved closer to the rotor until it lifts, and the field and gradient are again determined. The lifting field determines the magnetic moment or the magnetic susceptibility, and the relationship between the lift and rotation field is used to determine the rotation index. Usually these measurements are each made at a number of different field rotation frequencies. Magnetic susceptibilities do not show measurable change with field rotation frequency, but rotation indices can change significantly (either way) with increasing field rotation frequencies.

The magnetic field out from magnet rotors similar to the one in Fig. 1 can be found from the expression

$$
B=\frac{C}{e^{x / x_{0}}}
$$

where $C$ is the field at the rotor surface (Tesla); $x$ is the distance out from the rotor surface $(\mathrm{m})$ and $x_{0}$ is the depth of field (distance at which field reduces to $1 / e$ of its surface value) (m). 
The field gradient is the derivative of the above expression. Equation (3) may seem a very simplified expression for a complex magnet assembly, but calculated fields are always within actual field measurement error. Both the tangential fields (between the magnet poles) and the radial fields (above the magnet poles) follow the same expression, and, in a well-constructed magnet rotor assembly, are of equal magnitude (or at least within $10 \%$ ).

The magnetic mass susceptibility (for paramagnetic minerals) can be calculated using expression (4) [2]. The magnetic mass moment (SI units of $\mathrm{Wb} \mathrm{m} \mathrm{kg}^{-1}$ ) is almost the same, with the only difference being the absence of the field gradient term in the denominator.

$$
\left.k_{g}=\frac{\mu_{0} a\left(1-D_{f} / D_{p}\right)}{B_{l}\left(\partial B_{l} / \partial x\right)} \quad \text { (SI unit: } \mathrm{m}^{3} \mathrm{~kg}^{-1}\right)
$$

where $a$ is the acceleration due to gravity $\left(\mathrm{m} \mathrm{s}^{-2}\right) ; D_{f}$ is the specific gravity of the immersion fluid; $D_{p}$ is the specific gravity of the particle and $B_{l}$ is the lifting field (Tesla).

Equation (4), using the above method, is not meant to give an accurate susceptibility such as might be obtained with much more specialised equipment, and the accuracy depends on particle size, but the measurement can be routinely and easily carried out on tens or hundreds of individual particles as small as $50 \mu \mathrm{m}$, using the same equipment that would normally be used for magnetic separations.

Rotation index measurements are less precise, primarily because of variable particle shape. Paramagnetic particles with noncubic structure can rotate in a rotating field, especially if elongated or lamellae in shape, but for most paramagnetics the rotation is too weak to be of practical use. Most "rotatable" particles are ferromagnetic or at least contain ferromagnetic components as inclusions or exsolution regions, and therefore Eq. (5) [2] is written as ferromagnetic rotation index. The expression assumes that the ferromagnetic components are magnetically saturated at the rotation field, or, in other words, that the rotation field is greater than the critical field for domain wall motion.

$$
R_{f}=\frac{\mu_{0} d\left\{a\left(1-D_{f} / D_{p}\right)-\left(m_{m} / \mu_{0}\right)\left(\partial B_{r} / \partial x\right)\right\}}{2 m_{m} B_{r}} \quad \text { (dimensionless) }
$$


where $B_{r}$ is the rotation field (Tesla); $m_{m}$ is the mass magnetic moment $\left(\mathrm{Wb} \mathrm{m} \mathrm{kg}^{-1}\right)$ and $d$ is the particle diameter $(\mathrm{m})$.

As was mentioned above, the rotation index is based on the sine of the angle between particle magnetisation and the external field, and as such the rotation index should not measure as greater than one. In practice it can, because some particles are more rounded than the assumed cubic shape. The particle shape can be corrected for, either very approximately by an observation of the particle, or more precisely by measuring the rotation index over a range of field rotation frequencies and then using an expanded form of Eq. (2) to model the frequency response. Such correction would not normally be attempted because it would remove an important practical separation factor from the data.

\section{INTERPRETATION OF ROTATION INDEX AND MAGNETIC SUSCEPTIBILITY PLOTS}

Perhaps it would be more correct to plot rotation index against magnetic moment rather than against magnetic susceptibility, but magnetic susceptibility is retained here to avoid confusion because many particles contain elements of both types of magnetism, and most magnetic measurements in the mineral processing industry appear to be made in terms of susceptibility (using a variety of units). A paramagnetic particle will give a constant susceptibility no matter what the measuring field, but a calculated susceptibility on a saturated ferromagnetic particle will be high if measured in a low field and lower if measured in a high field.

The most useful representation of a set of susceptibility and ferromagnetic rotation index measurements, and one which is used below, is to use logarithmic axes for both values. If some measurements on paramagnetic particles give zero (or small negative) indices, these can be omitted or given small positive numbers (e.g., 0.0001) so as to obtain a log plot for the ferromagnetic particles.

When the external field required for rotation is weak compared to the anisotropy field in a particle, the theoretical rotation index should equal the sine of a quarter of the angle between successive magnetisation directions in the particle. If the particle contains only 
one type of ferromagnetic component, and varies from being completely ferromagnetic to containing only a small ferromagnetic component in a nonmagnetic matrix, the result is a constant rotation index across the range of susceptibilities (or magnetic moments) as shown in Fig. 2. The plots will be at different heights, depending on the angle between magnetisation directions.

For a particle with a single unidirectional magnetisation direction, the theoretical rotation index is 1 . This will only be the case for a permanent magnet or for a magnetised particle where the rotation field is below the critical field for domain wall motion. If a particle has a single bidirectional axis of magnetisation, the theoretical rotation index will be approximately 0.7 . This would be the case, for example, for a hexagonal particle with the magnetisation along the $c$ axis. If a particle has three directions of easy magnetisation at right angles (for example magnetite), the theoretical rotation index is approximately 0.4. For a hexagonal particle with three axes of magnetisation in the basal plane, the theoretical rotation index is 0.25 .

If the anisotropy field for a particle is of comparable strength to the external field, the maximum attainable angle between particle

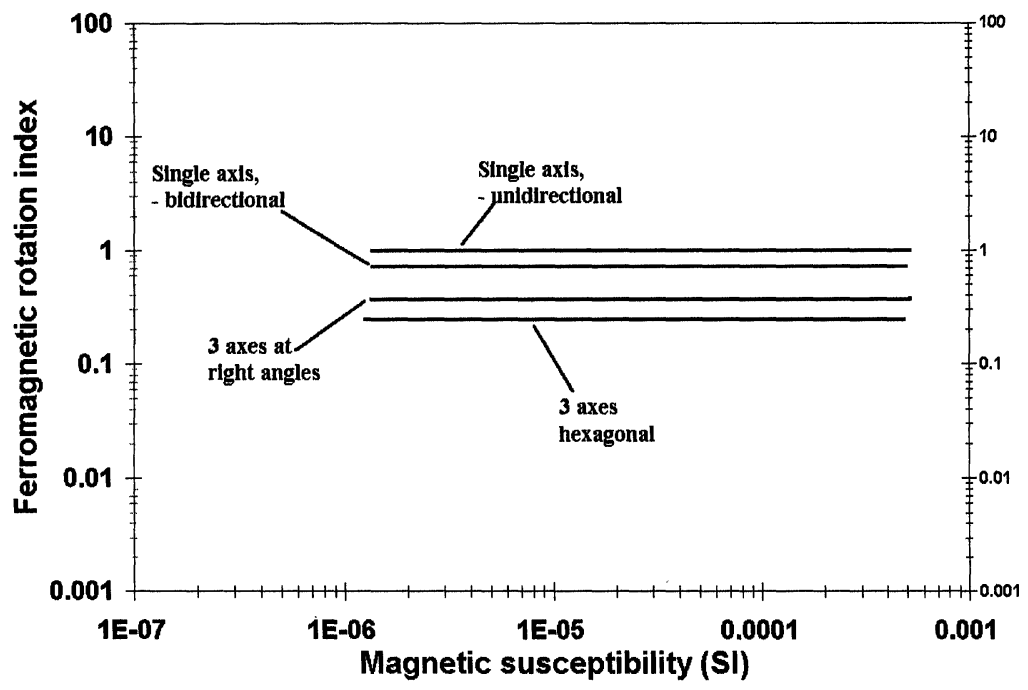

FIGURE 2 The effect of varying crystallographies. 
magnetisation and the external field will be smaller, and the measured rotation index will be correspondingly smaller. Therefore, in the absence of some knowledge of the anisotropy field, some caution is required when attributing a plot of rotation indices to a particular arrangement of magnetisation axes.

Paramagnetic particles can often contain ferromagnetic inclusions or components of varying proportions. The paramagnetic susceptibility sets a lowest susceptibility limit for the particles, with a decreasing proportion of the overall "susceptibility" being due to the ferromagnetic component with decreasing susceptibility. The plot then has the shape indicated in Fig. 3, with the height of the plot depending on the factors illustrated in Fig. 2.

The effect shown in Fig. 3 could also occur if there was a decrease in anisotropy field as the ferromagnetic component decreased within a nonmagnetic matrix. On the other hand, if ferromagnetic inclusions within a paramagnetic matrix become thinner as the total ferromagnetic component decreases, then shape anisotropy can reduce the slope of the right hand part of the trace in Fig. 3 and produce a sharper "turn-down" towards the paramagnetic susceptibility.

Occasionally a group of particles will plot as a vertical trace over a narrow susceptibility range. This could indicate a variation in the

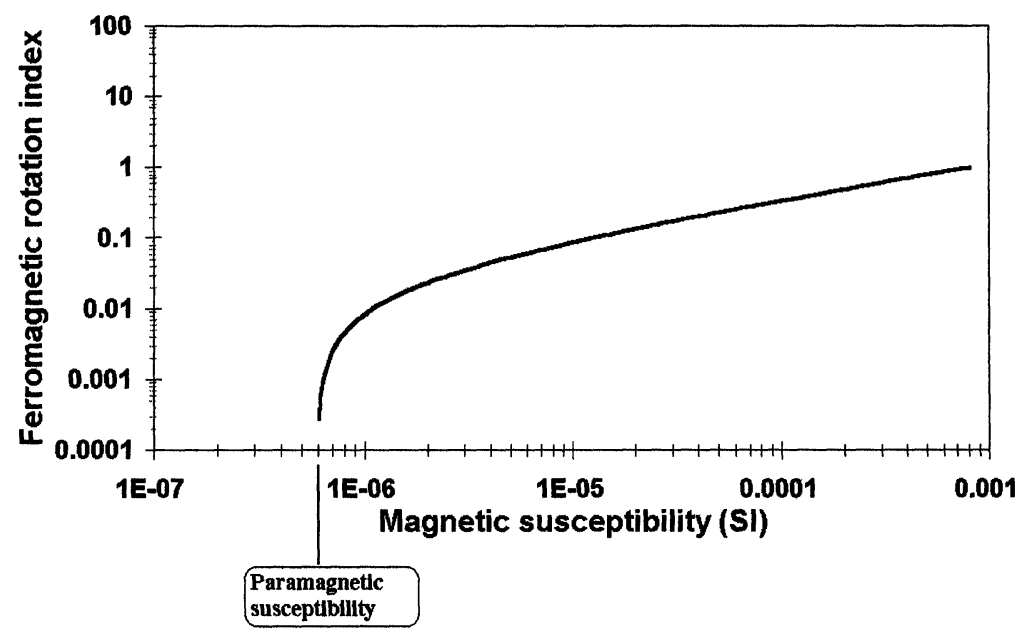

FIGURE 3 The effect of varying ferromagnetic content in a paramagnetic matrix. 
crystallography of the ferromagnetic components or a decrease in the anisotropy field within the ferromagnetic components, but no change in the quantity of the magnetic ions.

\section{INTERPRETATION OF ROTATION INDEX FREQUENCY RESPONSE}

There are two main factors that can affect the frequency response of the particle rotation index. Perhaps the most obvious is particle inertia. When a stationary particle is placed in a rotating magnetic field, the torque applied to the particle is not constant. If the external field is in the same direction as the particle easy magnetisation, the torque is zero. As the external field rotates away from the easy magnetisation direction the torque increases until the angle between the external field and the easy magnetisation direction is a quarter of the angle between successive magnetisation directions. As the angle increases further the torque decreases, and then changes direction as the external field approaches the next direction of easy magnetisation.

If the particle is going to rotate, it has to reach field rotation frequency shortly after maximum torque is applied. If a particle only just rotates at one field frequency and then the field rotation frequency is increased, the particle can no longer commence rotation at the original field strength simply because it can no longer reach field rotation frequency in the shorter available time. It is necessary to increase the external field so as to increase the angular acceleration of the particle. The measured rotation index then decreases as shown in Fig. 4.

The second factor is domain wall motion. For some minerals, such as natural magnetite, the field strength required for domain wall motion is only just below the field strength required for particle rotation. When this is so, domain wall travel times across a particle can become comparable to field rotation periods. This has the effect of increasing the maximum attainable angle between particle magnetisation and the rotating external field, because the particle magnetisation cannot respond quickly enough. As the external field rotation frequency is increased, the particle will appear to require lower fields to commence rotation, giving an apparent increase in rotation index. 


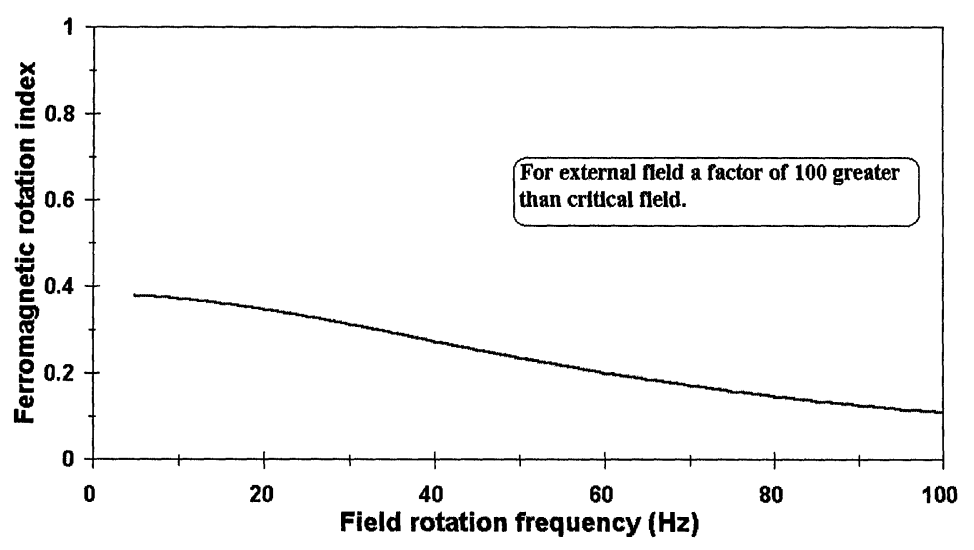

FIGURE 4 The inertia effect on rotation index frequency response.

Above a maximum field rotation frequency the particle begins to demagnetise, requiring higher fields to commence rotation, and decreasing the measured rotation index again. The effect of slow domain wall velocity on the rotation index frequency response is shown in Figs. 5(a) and (b). The minimum external field required to rotate the particle $\left(H_{\text {sep }}\right)$ is different at each field rotation frequency, and is given here only for a field rotation frequency of $10 \mathrm{~Hz}$.

\section{SOME EXAMPLES AND USES OF ROTATION INDEX AND SUSCEPTIBILITY PLOTS}

Figure 6 shows a plot of rotation indices and susceptibilities for some magnetic particles. Some of the horizontal variation in susceptibility is probably due to composite particles in this case. However the vertical spread in rotation indices at the same susceptibility value has to have another explanation. Microprobe analysis of the measured grains shows no clear compositional explanation for it, and particle shape effects cannot produce such a wide distribution, so the answer probably lies in a combination of differing domain wall velocities, differing anisotropy fields and differing crystal sizes within particles.

Figure 7 shows a rotation index and susceptibility plot for chrome spinel particles. The particles referred to as $\mathrm{Pt} 2$ and $\mathrm{Pt} 3$ are associated 
(a)

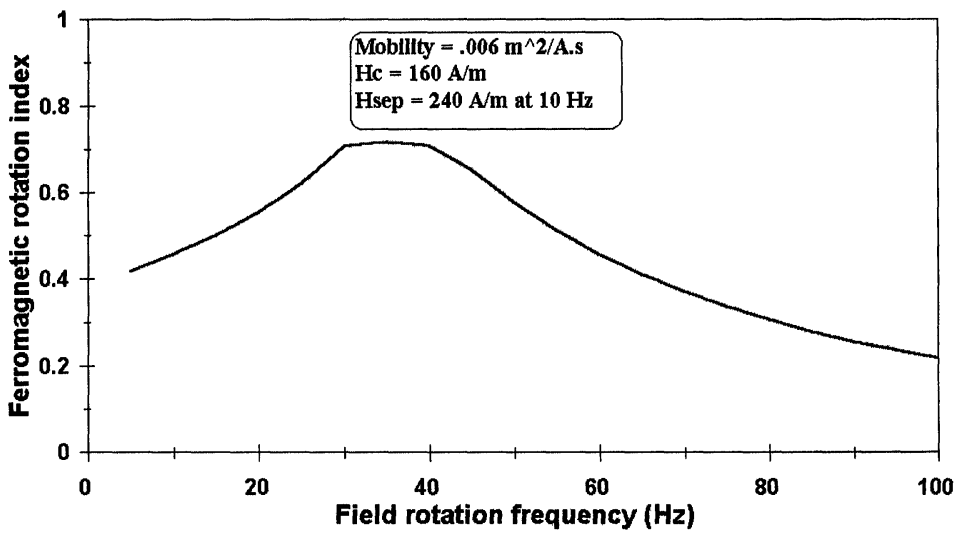

(b)

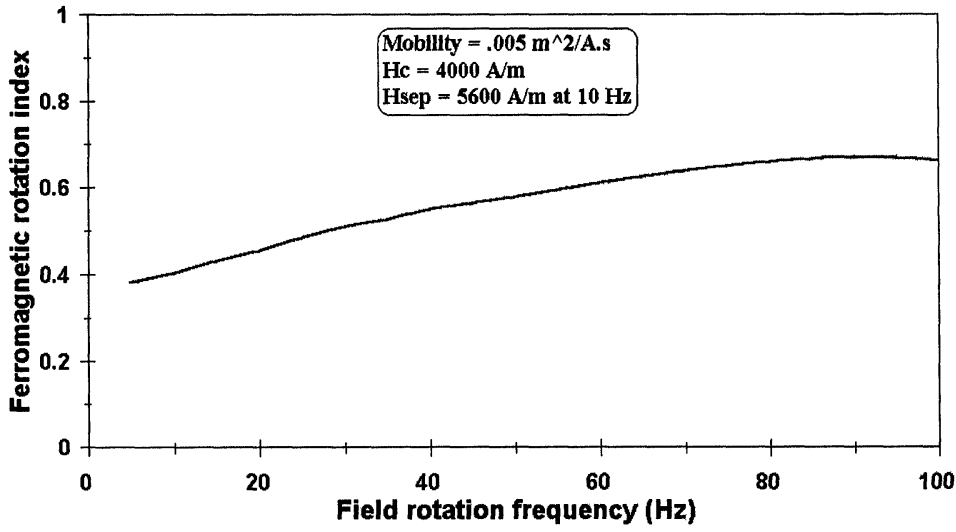

FIGURE 5 (a) Domain wall velocity effect on rotation index frequency response for rotation field very close to critical field. (b) Domain wall velocity effect on rotation index frequency response for rotation field further from critical field.

with platinum ore-bodies. The Pt 2 chrome spinels come from a rock with a platinum content of $0.9 \mathrm{ppm} \mathrm{Pt}$, while the Pt 3 chrome spinels come from a rock at a different location that assayed at $0.2 \mathrm{ppm}$ platinum. The "basalt" chrome spinels come from north-east Tasmania, from a stream draining off a Tertiary basalt.

The "high-platinum" (Pt3) chromites in Fig. 7 form a restricted plot that follows the pattern of Fig. 3, indicating ferromagnetic components within a paramagnetic matrix. The vertical dispersion (width) of this 


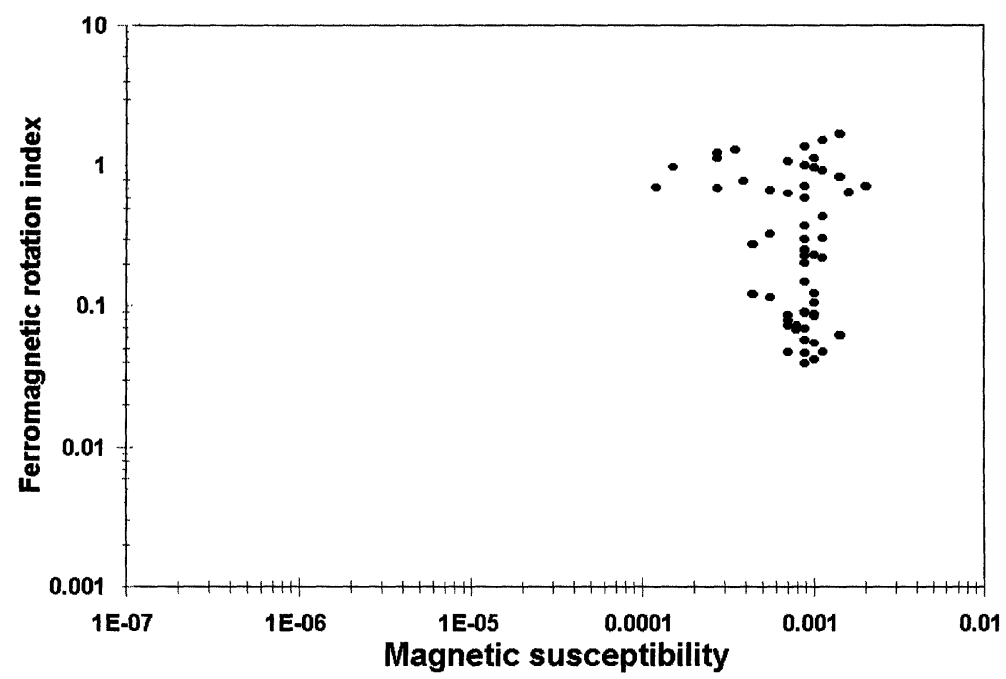

FIGURE 6 A plot of some rotation indices and magnetic susceptibilities for magnetite.

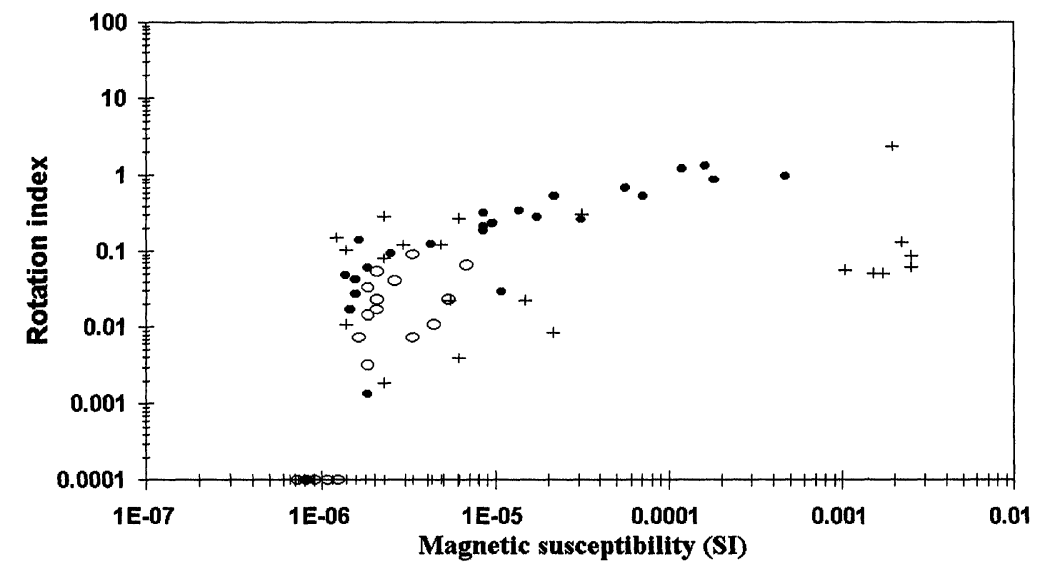

\section{- Pt $3 \circ$ Pt $2+$ Basalt}

FIGURE 7 A plot of some rotation indices and magnetic susceptibilities for chrome spinel. 
plot is well within expected measurement error due to variable particle shape. The height of the plot suggests only a single axis of easy magnetisation, and microprobe mapping of a polished surface through one grain shows the presence of elongated regions sufficiently high in $\mathrm{Fe}^{3+}$ for an intermediate state between inverted and normal spinel.

The "low platinum" (Pt2) chromites tend to be much lower in susceptibility and may be indicating two different magnetic structures, one similar to the Pt 3 chromites and the other indicating more than one axis of easy magnetisation. These chromites do not reach to the higher rotation indices of the $\mathrm{Pt} 3$ chromites.

The "basalt" chrome spinels in Fig. 7 more definitely indicate two quite distinct ferromagnetic structures, with grains tending to have one or the other but not both (few grains in between the two traces). One of the structures plots above the Pt 3 chromites at low susceptibilities, and may be indicating the presence of shape anisotropy in the ferromagnetic components. The second structure extends to very high susceptibilities (equal to those for magnetite), but has very low rotation indices that indicate either a low anisotropy field or angles of considerably less than $90^{\circ}$ between magnetisation directions, or both. However, there is no obvious compositional difference between the two types of chromites here. Whatever the reason for the low rotation indices, the two traces indicate quite different magnetic structures, and the fact that some particles do not appear to contain both structures points to a different origin for the two types of chromite particles within the basalt.

In addition to suggesting the properties of ferromagnetic inclusions, the data in Fig. 7 also suggests that chrome spinel particles formed under different conditions may have characteristic magnetic properties that can be identified from a plot of susceptibility and rotation indices. Therefore plots of particle magnetic characteristics could find a use as an exploration tool. However, before this can be realised, there needs to be a great deal more experience with the plots. Unfortunately the data on mineral particle rotation is very limited at this stage.

Allen [3] illustrated the use of rotation index and susceptibility measurements to design a separation method for the concentration of picro ilmenites from ilmenites in the diamond exploration industry. Once a representative set of particle measurements has been obtained, the values can be used to simulate the effects of various combinations 
of attraction and rotation separation before designing a real magnetic separation process.

\section{CONCLUSIONS}

Measurements of individual particle magnetic susceptibility and rotation index can be made routinely on the same equipment used for Rotating Magnetic Field (RMF) separation.

Plots of the rotation index and susceptibility measurements can indicate the magnetic structure of the ferromagnetic components present in the particles, and perhaps could, at least in the case of chromite, be used as indicators of mineral genesis. The plots can also indicate the most effective magnetic separation method (rotation or attraction) or combination of rotation and attraction methods.

At present magnetic measurements on individual particles have only been made for ilmenite and chrome spinel, with a few measurements only on magnetite. This has so far limited the application of RMF rotation separation to these minerals. No compositionally correlated measurements have yet been made on other minerals of interest for magnetic separation, such as tantalite, wolframite, siderite, monazite, xenotime, pyrrhotite, etc.

\section{References}

[1] J.C. Maxwell (1873). A Treatise on Electricity and Magnetism, Vol. I and II, Macmillan and Co., Oxford.

[2] N.R. Allen (1999). Rotating magnetic field separation of minerals, PhD Thesis (unpublished), University of Tasmania, Hobart.

[3] N.R. Allen. Mineral separation by particle rotation. In: Magn. Electr. Sep. (in print). 